\title{
Clinical comparison of Sentinel, a novel blood culture system, with radiometric Bactec 460 and Isolator 10 in the detection of streptococcal and anaerobic bacteraemias
}

\author{
N A Hutchinson, D C Shanson, T Malins
}

Department of

Medical Microbiology,

Charing Cross and

Westminster Medical

School, 17 Horseferry

Road, London SW1P

2AR

N A Hutchinson

D C Shanson

Department of Oral and Maxillofacial

Surgery, Westminster

Hospital

T Malins

Correspondence to:

Dr DC Shanson

Accepted for publication

17 November 1991

\section{Abstract}

Aims: To compare the performance of the Sentinel blood culture system with two other systems for the recovery of streptococci and anaerobes.

Methods: Blood cultures were taken from 55 patients one to two minutes after dental extraction. The samples were tested by the radiometric Bactec 460; the Isolator 10 , which works by lysis centrifugation; and Sentinel, a fully automated system, which detects bacterial growth by changes in the voltage between two electrodes in the media. Positive samples were subcultured and streptococci and anaerobes were further identified. Terminal subcultures were performed on all negative samples.

Results: Sentinel was comparable with

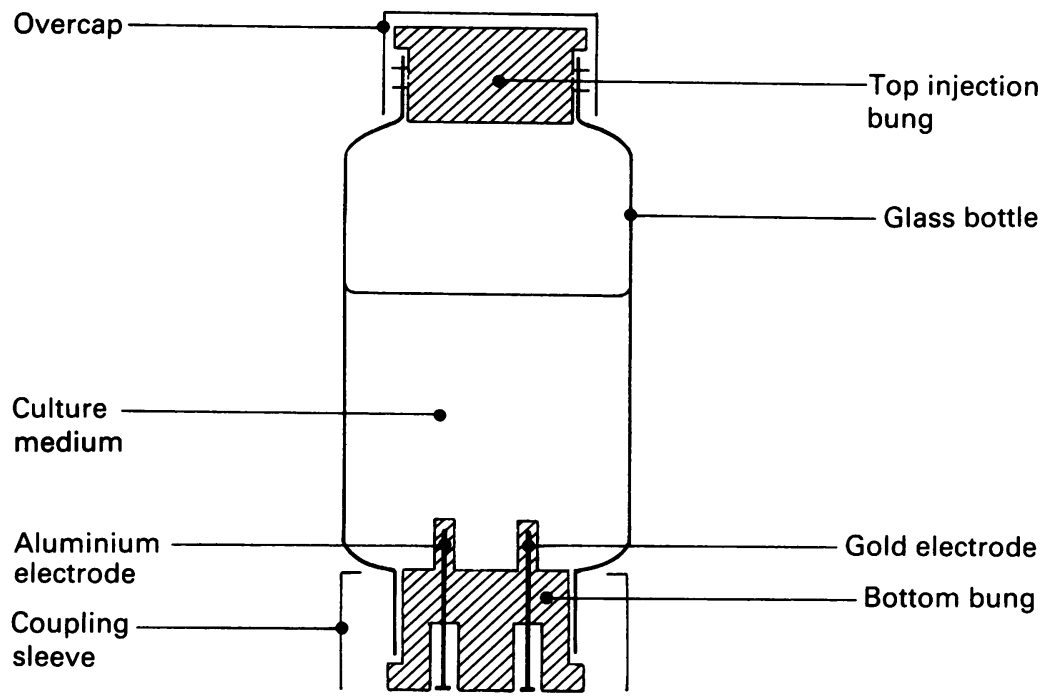

Indexed into instrument

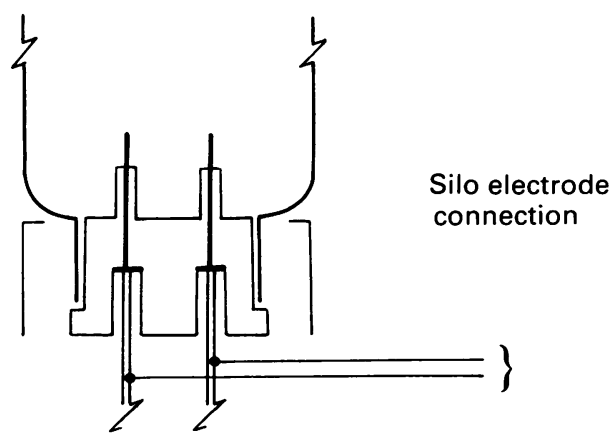

Figure 1 Bottle components.
Bactec, with Sentinel recovering 20 streptococci and 14 anaerobes; Bactec isolated 26 streptococci and 15 anaerobes. The recovery of Streptococcus sanguis was significantly better from Bactec than Sentinel. The Isolator 10 recovered significantly fewer streptococci and anaerobes than either Bactec or Sentinel. Sentinel was noticeably quicker in detecting anaerobes than Bactec 460. However, there was no comparable difference with streptococci. Contaminants were recovered from $10 \%$ of Isolator $10,2 \cdot 7 \%$ of Bactec, and $7 \cdot 2 \%$ of Sentinel bottles.

Conclusions: Sentinel and Bactec 460 were broadly comparable in the detection of viridans streptococci and oral anaerobes, and both systems were significantly better than the Isolator 10. However, the prototype Sentinel, was significantly poorer than Bactec for the recovery of $S$ sanguis.

Reliable recovery of viridans streptococci from blood cultures is important for the microbiological diagnosis of endocarditis and streptococcal septicaemia in immunocompromised patients. Techniques that may facilitate this can be conveniently tested in dental patients who have predictable bacteraemias following extraction.

Sentinel (Difco Laboratories Ltd, East Molesey, Surrey, England) is a new, fully automated blood culture system driven by a Tandon PCA 20 microcomputer which continuously monitors the samples. Sentinel consists of incubation modules, called drawers, which contain 80 "silos". These are thermostatically controlled, self-contained units into which the culture bottle is inserted. On insertion two electrodes (one aluminium, one gold plated) are indexed up into the media from the bottle's base bung (fig 1) and a voltage of about $0.5 \mathrm{~V}$ is generated. The voltage is produced by the aluminium electrode slowly dissolving, liberating $\mathrm{Al}^{3+}$ ions into the media, and the resulting electrons passing through the electrical circuits in the base bung to the gold electrode where they reduced chemical compounds in the media. In the aerobic Sentinel bottle the major reducible species is dissolved oxygen; bacterial growth takes up this oxygen thus making it unavailable for removal of the electrons at the gold electrode and so the voltage declines. The computer records the change, and determines 

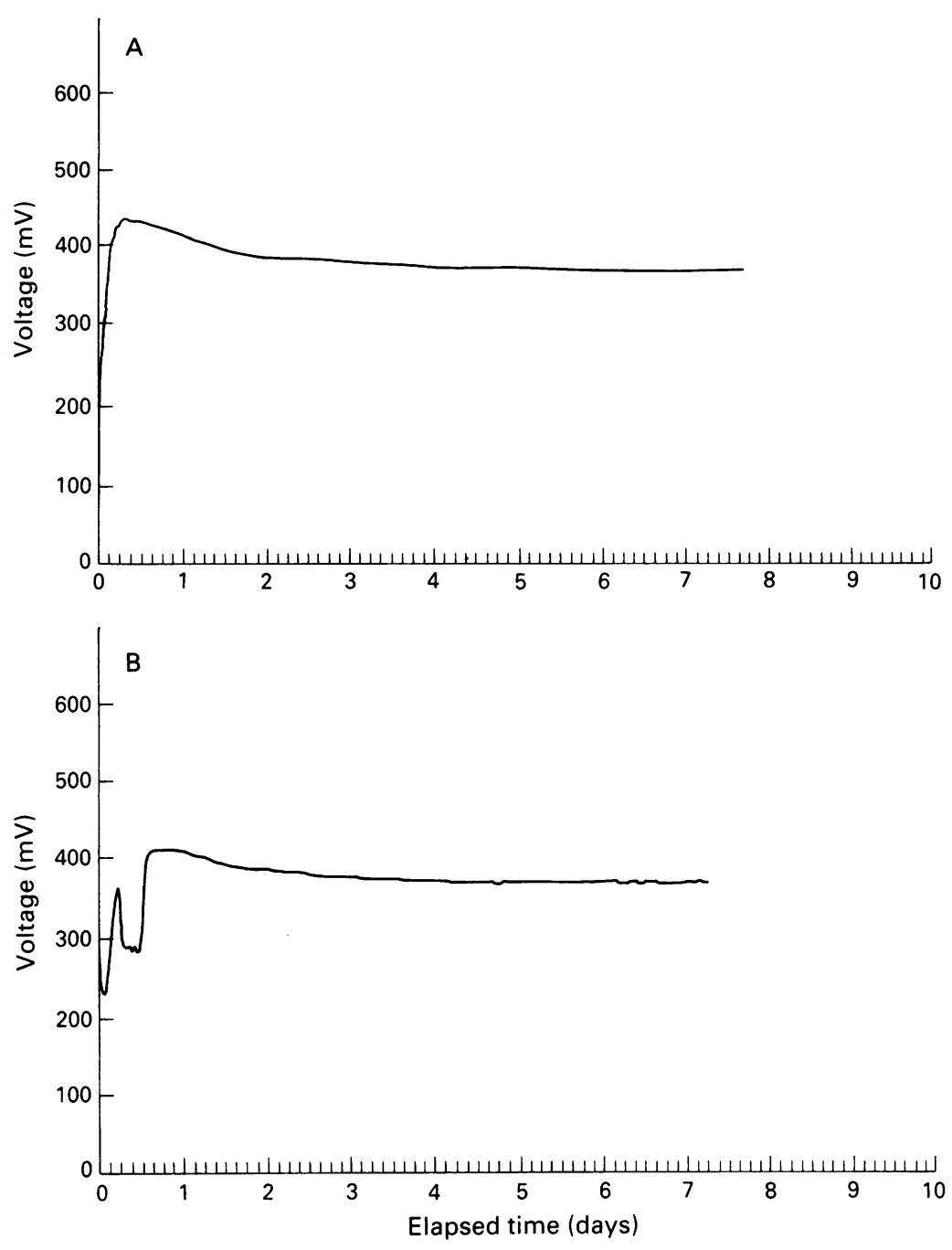

Figure 2 Signal from anaerobic Sentinel bottle with sterile blood.

whether it is significant using an algorithm. In the anaerobic bottle the electron accepting system is oxygen free and the bacteria reduce an alternative chemical electron acceptor which produces a drop in voltage.

Sentinel is of particular interest as a "load on and walk away" automated system which saves labour by "flagging up" suspect positive results which need further identification.

In 1990 Sentinel was compared with radiometric Bactec 460 and the Isolator 10 for the detection of viridans streptococci and nonsporing anaerobes. This is achieved by inoculating blood collected from patients undergoing dental extraction. Earlier reports by Shanson ${ }^{1-3}$ show that streptococci can be isolated from between $32 \%$ and $64 \%$ and nonsporing anaerobes from between $25-47 \%$ of blood cultures taken one to two minutes after extraction.

\section{Methods}

\section{BLOOD CULTURE MEDIA}

The Bactec culture phials used were the $6 \mathrm{~B}$ (enriched soybean-caesin digest broth) for aerobic culture and the $7 \mathrm{D}$ (pre-reduced enriched soybean caesin digest) for anaerobic culture. The Isolator 10 tube contains purified saponin, polypropylene glycol, sodium polyanotholsulfonate and EDTA. The Sentinel system has two bottles, each with $50 \mathrm{ml}$ of media, and both contain liquoid. The anaerobic Sentinel medium is based on Difco thiol broth.

\section{PATIENT BLOOD COLLECTION METHODS}

Fifty five adults undergoing dental extraction, under general or local anaesthetic, at Westminster Hospital were entered into the trial. Those who were known to have heart lesions and to be at risk of infective endocarditis, and those who had received antibiotics up to one week before surgery were excluded, and prophylaxis against local infection was briefly delayed until after blood culture collection. Thirty $\mathrm{ml}$ of blood was drawn one to two minutes after the first tooth had been extracted. From this, $5 \mathrm{ml}$ was aseptically inoculated into each of the two Bactec and two Sentinel bottles and $10 \mathrm{ml}$ into the Isolator tube. The order in which the media were inoculated was random.

\section{PROCESSING OF BLOOD CULTURES}

On arrival in the laboratory all cultures were processed immediately. The Bactec phials were incubated at $37^{\circ} \mathrm{C}$ with the aerobic bottles agitated for the first 48 hours. The bottles were tested twice on days 1 and 2 and once on days 3 to 7 . After seven days terminal subcultures were performed. The Sentinel bottles were entered into the system and inserted into silos. The aerobic bottles are permanently vented via a custom-made venting unit. The aerobic bottles gave a fairly consistent signal of C.500 mV with sterile human blood (fig 2A). The anaerobic bottles gave a more complex pattern with a longer development to a steady voltage at C. $350-400 \mathrm{mV}$ (fig 2B). There were, however, some irregularities in the voltage associated with blood cells settling in the first six to 12 hours. Therefore; the computer was programmed not to flag a sample as positive within the first 12 hours after insertion. If Sentinel flagged a sample as positive it was removed from the system and subcultured. Figs $3 A$ and $B$ give examples of the changes in voltage that occur in positive aerobic and anaerobic bottles. After seven days the remaining unflagged bottles were removed from the system and terminal subcultures performed. The Isolator 10 tubes were immediately centrifuged on arrival at $3000 \times g$ for 30 minutes. The supernatant was removed using an Isostat supernatant pipette. The remaining deposit was mixed vigorously and then evacuated from the tube using an Isostat concentrate pipette and then inoculated onto two blood agar, one chocolate agar, and one Kanamycin blood agar plates. One blood agar and the chocolate agar plate were incubated at $37^{\circ} \mathrm{C}$ in $5 \%$ carbon dioxide for 48 hours and examined at 24 and 48 hours. The other blood agar and Kanamycin blood agar plates were incubated at $37^{\circ} \mathrm{C}$ in the anaerobic cabinet for six days and examined daily. Cultures from positive samples and terminal subcultures from the Bactec and Sentinel bottles were inoculated as for the Isolator subcultures 

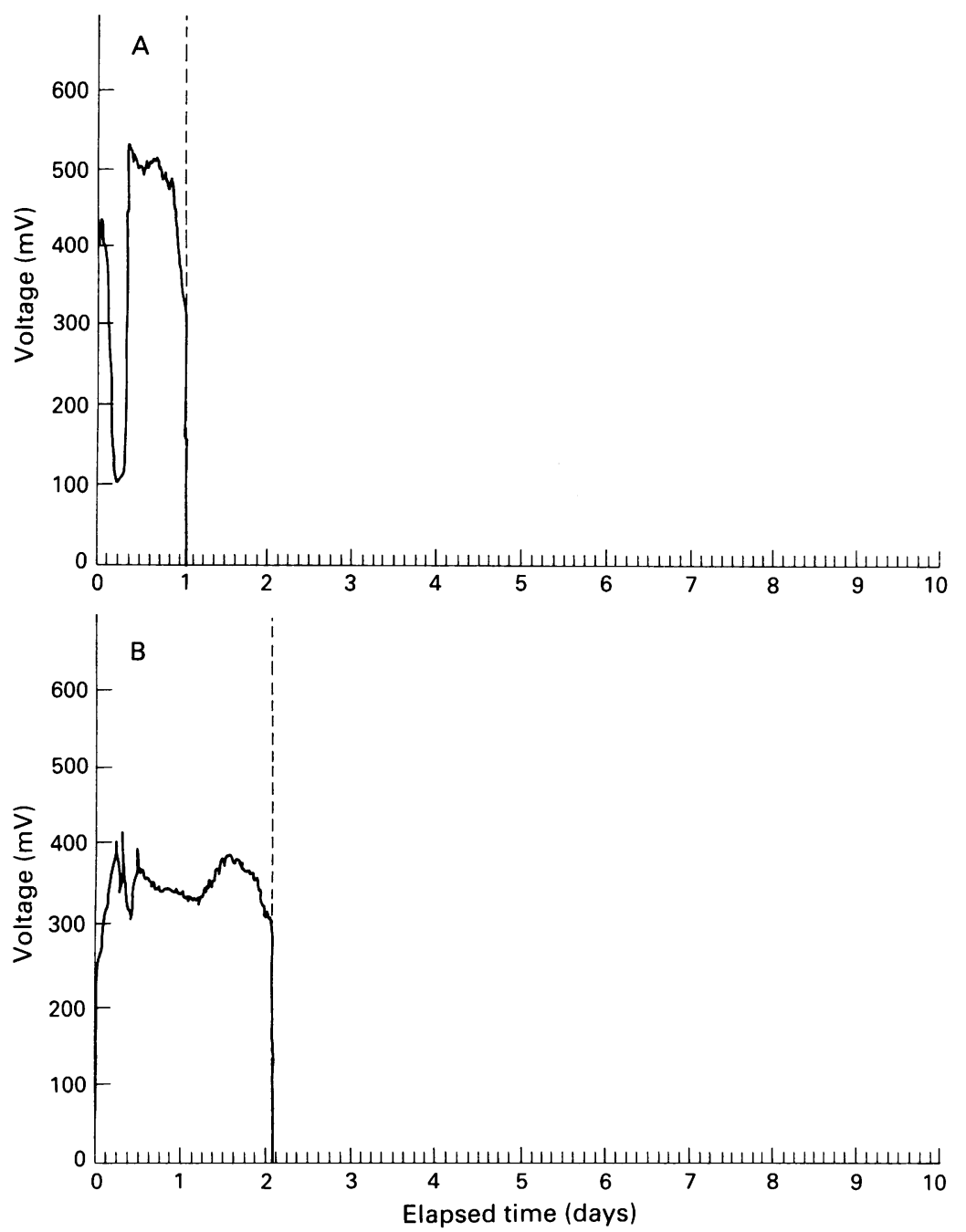

Figure 3 Signal from positive anaerobic Sentinel bottle.

with the exception that the anaerobic plates were incubated for five days.

IDENTIFICATION OF ISOLATES

Gram positive cocci that grew aerobically and were catalase negative were identified as streptococci and identified further with the API 20 streptococcal identification test (Biomèrieux UK Ltd, Grafton House, Grafton Way,

Table 1 Comparison of three blood culture systems for isolation of streptococci

\begin{tabular}{|c|c|c|c|}
\hline Case no & Bactec & Sentinel & Isolator 10 \\
\hline $\begin{array}{l}1 \\
2 \\
3 \\
4 \\
5 \\
6 \\
7 \\
8 \\
9 \\
10 \\
11 \\
12 \\
13 \\
14 \\
15 \\
16 \\
17 \\
18 \\
19 \\
20 \\
21 \\
22 \\
23 \\
24\end{array}$ & $\begin{array}{l}S \text { sanguis } \\
S \text { milleri } \\
S \text { sanguis }+S \text { milleri } \\
S \text { mutans } \\
S \text { sanguis } \\
S \text { sanguis } \\
S \text { mitis } \\
S \text { sanguis } \\
S \text { mitis } \\
S \text { sanguis } \\
S \text { morbillorum } \\
S \text { mitis } \\
S \text { milleri }+S \text { sanguis } \\
S \text { sanguis } \\
S \text { sanguis }+S \text { morbillorum } \\
S \text { sanguis } \\
S \text { sanguis }+S \text { mitis } \\
S \text { sanguis } \\
S \text { mitis }+S \text { sanguis } \\
S \text { morbillorum (TS only) } \\
S \text { sanguis }\end{array}$ & $\begin{array}{l}S \text { sanguis }+S \text { mitis } \\
S \text { milleri } \\
S \text { milleri (TS only) } \\
S \text { sanguis } \\
S \text { mitis } \\
S \text { sanguis } \\
S \text { mitis }+S \text { milleri } \\
S \text { mitis }\end{array}$ & $\begin{array}{l}S \text { sanguis } \\
S \text { sanguis } \\
S \text { mitis } \\
S \text { milleri }\end{array}$ \\
\hline
\end{tabular}

TS = Terminal subculture.
Table 2 Differences in time to detection in nine cultures yielding identical streptococci in Sentinel and Bactec systems

\begin{tabular}{ll}
\hline & No of isolates \\
\hline $\begin{array}{l}\text { Sentinel and Bactec within two hours } \\
\text { Sentinel greater than two hours faster } \\
\text { than Bactec }\end{array}$ & 1 \\
$\begin{array}{l}\text { Bactec greater than two hours faster } \\
\text { than Sentinel }\end{array}$ & 4 \\
\hline
\end{tabular}

Basingstoke, Hampshire). Suspected anaerobes were subcultured aerobically and anaerobically for up to five days. Isolates that grew only anaerobically and were sensitive to metronidazole were confirmed as anaerobes. These were further identified on colonial morphology, reaction to Gram stain, and antibiotic sensitivity pattern. The sensitivity tests were performed with an anaerobe identification MASTRING (Diamed Diagnostics Ltd, Bootle, Merseyside) that included penicillin 2 units, erythromycin $60 \mu \mathrm{g}$, rifampicin $15 \mu \mathrm{g}$, colistin $10 \mu \mathrm{g}$, kanamycin $1000 \mu \mathrm{g}$, and vancomycin $5 \mu \mathrm{g}$ discs.

The results were analysed using "the comparison of proportions" and significance given at the $5 \%$ level.

\section{Results}

Streptococcal species were isolated in $24(44 \%)$ sets of blood cultures (table 1). Sentinel isolated 20 streptococci from 15 patient samples; Bactec isolated 26 streptococci from 20 samples and the Isolator 10 recovered only five streptococci. Statistical analysis shows that although Bactec isolated more streptococci from more samples than Sentinel, the difference was not significant. Both Sentinel and Bactec, however, recovered significantly more streptococci than the Isolator 10 .

Sentinel and Bactec isolated the same streptococcal species in nine blood culture sets. In three cases Sentinel isolated a different streptococcus in addition and Bactec isolated an additional streptococci in two cases. In two patient sets both Sentinel and Bactec recovered an additional streptococcus as well as a common isolate, and from four cases Sentinel and Bactec recovered completely different streptococci. In eight patient samples the only blood culture system to detect streptococci was the Bactec 460 (in six of these the other two systems failed to recover $S$ sanguis). Streptococci were only isolated by Sentinel from a further two cases and in one sample the Isolator 10 was the only system to isolate a streptococcus.

In the nine cases where Sentinel and Bactec isolated the same streptococcal species there was no comparable difference in pathogen

Table 3 Numbers of isolates from each blood culture system

\begin{tabular}{llll}
\hline & Bactec & Sentinel & Isolator \\
\hline S sanguis & 14 & 5 & 2 \\
S mitis & 5 & 9 & 2 \\
S milleri & 3 & 5 & 1 \\
S mutans & 1 & & \\
S morbillorum & 3 & 1 & \\
Aerococcus viridans & & & \\
\hline
\end{tabular}


Table 4 Comparison of each system for detection of anaerobes

\begin{tabular}{llll}
\hline Case No & Bactec & Sentinel & Isolator 10 \\
\hline 1 & & B ureolyticus & \\
2 & B oralis & B ureolyticus & \\
3 & B ureolyticus & B oralis & B oralis \\
4 & B oralis & Veilonella sp & \\
5 & Veilonella sp & Anaerobic cocci & \\
6 & B melanogenicus & B ureolyticus & B melanogenicus \\
7 & Anaerobic cocci & Anaerobic cocci & \\
8 & Veilonella sp (TS) & B melanogenicus & \\
9 & B melanogenicus & & B melanogenicus \\
10 & B ureolyticus & Anaerobic cocci & \\
11 & Anaerobic cocci & Anaerobic cocci & \\
12 & B melanogenicus & Anaerobic cocci & Anaerobic cocci \\
13 & Anaerobic cocci & Anaerobic cocci & \\
14 & Beilonella sp & B melanogenicus \\
15 & B melanogenicus & Anaerobic cocci & Anaerobic cocci \\
16 & Anaerobic cocci & & \\
18 & & & \\
19 & & & \\
21 & 22 & &
\end{tabular}

detection time (table 2). Streptococci recovered from terminal subculture indicates a failure of detection by the culture system and this occurred once with both Sentinel and Bactec. Table 3 gives the number of particular species isolated from each blood culture system. Bactec was significantly better at the recovery of $S$ sanguis than Sentinel.

Anaerobic organisms were isolated from 23 $(42 \%)$ blood culture sets (table 4 ). Sentinel isolated 14 anaerobes, Bactec 15, and the Isolator 10.7. There was no significant difference in the comparison of Sentinel with Bactec for the recovery of oral anaerobes. Both Sentinel and Bactec were significantly better than the Isolator $10(p<0.05)$. Sentinel and Bactec isolated the same anaerobe in six cases and in two sets recovered different anaerobes. Comparing Sentinel with the Isolator 10, in three cases both systems detected the same organism and different anaerobes in two cases. The six cases in which the same anaerobe was isolated by both Sentinel and Bactec can be used to compare each system for their speed in pathogen detection (table 5). In five cases Sentinel detected the anaerobe more promptly than Bactec, and in three of these the difference was greater than 24 hours. There were no false negative results with Sentinel and only one with Bactec.

Table 6 gives the number of the particular anaerobes isolated by each system. Bacteroides melaninogenicus was the anaerobe more frequently isolated by both Bactec and the Isolator 10 systems but not at all by Sentinel.

Organisms considered to be of doubtful importance, such as diphtheroid species, Bacillus species, and staphylococci, were found in $2.7 \%$ of Bactec cultures, $8 \%$ of Sentinel, and $10 \%$ of the Isolator 10 .

Table 5 Differences in time to detection in six cultures yielding identical anaerobes in Sentinal and Bactec systems

\begin{tabular}{ll}
\hline & $\begin{array}{l}\text { No of anaerobic } \\
\text { isolates }\end{array}$ \\
\hline $\begin{array}{l}\text { Sentinel and Bactec within two hours } \\
\text { Sentinel greater than two hours faster } \\
\text { than Bactec }\end{array}$ & 1 \\
$\begin{array}{l}\text { Sentinel greater than } 24 \text { hours faster than } \\
\text { Bactec }\end{array}$ & 2 \\
$\begin{array}{l}\text { Bactec greater than two hours faster than } \\
\text { Sentinel }\end{array}$ & - \\
\hline
\end{tabular}

Table 6 Numbers of anaerobic isolates detected

\begin{tabular}{llll}
\hline & Bactec & Sentinel & Isolator \\
\hline B oralis & 2 & 2 & 1 \\
B ureolyticus & 2 & 3 & \\
B melanogenicus & 5 & 2 & 4 \\
Veilonella sp & 1 & 7 & 2 \\
Anaerobic cocci & 4 & 7 \\
\hline
\end{tabular}

\section{Discussion}

The overall results from this clinical trial indicate that the performance of the Sentinel system was comparable with that of radiometric Bactec 460 in its isolation of viridans streptococci and oral anaerobes. Sentinel isolated 20 streptococci from 15 blood culture sets and 14 anaerobes; Bactec recovered 26 streptococci from 20 samples and 15 anaerobes. Sentinel recovered significantly fewer $S$ sanguis organisms and this may possibly have been due to killing of the organism after growth started because of too much glucose $(5 \mathrm{~g} / \mathrm{l})$ in the medium and an untoward drop in $\mathrm{pH}$. Difco have subsequently modified the aerobic Sentinel media to a reduced glucose concentration $(2.5 \mathrm{~g} / \mathrm{l})$, and this should be more satisfactory for the recovery of $S$ sanguis. This has obvious implications for the diagnosis of endocarditis of which $S$ sanguis is an important pathogen.

The Isolator 10 recovered significantly fewer streptococci $(n=5)$ and anaerobes $(n=7)$ than either Bactec or Sentinel. Similar results were obtained by Brannon and Kiehn, ${ }^{4}$ who reported that the radiometric Bactec recovered significantly more streptococci and anaerobes than the Isolator 10 . There was also a significant difference in the isolation of viridans type streptococci. However, Kellog et $a l^{5}$ found no significant difference between Bactec and Isolator 10 for the detection of streptococci but did report that Bactec was significantly better for the detection of anaerobes.

Both Bactec and Sentinel systems had one false negative result, but Sentinel had four false positive samples which gave a positive flagging but failed to grow on subculture. Notably, with two of these samples Bactec yielded a $S$ sanguis. A large proportion of the bacteraemias detected were polymicrobial; this is to be expected as the microbial flora of the oral cavity is heterogeneous.

The bacteraemia caused by dental extraction is low level-that is, only a few organisms enter the bloodstream-and this may be one reason why species appear in some $5 \mathrm{ml}$ blood cultures and not others.

We thank Difco, the bacteriology staff at Westminster Hospital, and Mrs M Mountain for secretarial support.

1 Shanson DC, Thomas FD, Johnstone D. Improving detection of 'viridans streptococcus' bacteraemia by adding sodium polyanothol sulphate to blood cultures. $J$ Clin Pathol 1985;38:1346-8.

Shanson DC, Akash S, Harris M. Clinical comparison of glucose broth with nutrient broth blood cultures for the detection of streptococcus viridans bacteraemia. $J \mathrm{Med}$ Microbiol 1983;16:497-9.

3 Shanson DC, Moule I, Tadayon M. Clinical comparison of anaerobic blood culture media for detecting bacteraemia due to viridans streptococci and oral anaerobes. $J \mathrm{Med}$ Microbiol 1985;19:187-93.

4 Brannon P, Kiehn TE. Large scale clinical comparison of ysis centrifugation and radiometric systems for blood culture. J Clin Microbiol 1985;22:951-4.

5 Kellog JA, Manzella JP, McConville JH. Clinical laboratory comparisons of the $10 \mathrm{ml}$ Isolator blood culture system with Bactec Radiometric blood culture media system Microbiol 1984;20:618-23. 